\title{
Structure and Symmetrization of Hydrogen Bonding in Ices VIII and X at High Pressure: A Density Functional Theory Approach
}

\author{
Nurapati Pantha and Narayan Prasad Adhikari \\ Central Department of Physics, Tribhuvan University, Kathmandu, Nepal. \\ Emai-:npadhikari@tucdp.edu.np; npadhikari@gmail.com
}

\begin{abstract}
We study the change in structural properties of ice by taking initial structure of "ice VIII", with space group symmetry I41/amd, as a function of elevating pressure up to $120 \mathrm{GPa}$ in density-functional theory (DFT) level of calculations implemented by Quantum ESPRESSO package. Consistent with the standard laws of thermodynamics, our calculations show that the physical size (volume and cell parameters) of the unit cell compresses monotonically on increasing pressure. We also compare our DFT results of these parameters with the available experimental values performed at finite temperature. The comparison shows good agreement between the quantities, within $5 \%$, with slightly higher experimental values. At $100 \mathrm{GPa}$ of pressure, hydrogen atom comes exactly at the midpoint of two boneded oxygens, called hydrogen-bonded symmetrization, which at low pressure remains nearby one of the oxygens. This symmmetrized structure is characterized by a new phase of the system known as "ice X" and the boundary pressure, $100 \mathrm{GPa}$, defines the transition pressure $\left(\mathrm{P}_{0}\right)$ for changing phase from "ice VIII" to "ice $\mathrm{X}$ ". The transition pressure $\left(\mathrm{P}_{0}\right)$ of the present work agrees well within $2 \%$ of previously reported results.
\end{abstract}

Keywords: Quantum Espresso, Ice VIII, Ice X, DFT

\section{INTRODUCTION}

Ice is a simple compound and chemically formed by two atoms of the hydrogen and one atom of oxygen. In liquid form, we see the same composition as water which has numerous applications in daily life and also in scientific arena as solvent, solute and biological molecule (Adhikari et al. 2011). Ice and/or water is physically dominant in the universe and closely related to the many disciplines of the scientific world. Pure ice as a solid and its mixture in fluid with other compounds like $\mathrm{CH}_{4}, \mathrm{CO}_{2}$ and $\mathrm{NH}_{3}$ at extreme conditions have the great implications in planetary science (Lee \& Scandolo, 2011; Cavazzoni et al. 1999) and condensed matter physics/chemistry (Sloan \& Koh, 2007; Hemley et al. 1987).

In spite of its simplicity in atomic composition, ice has complex and multiple phases (Kush et al. 1984). Depending on varying environmental conditions, it is found in more than a dozen of different phases (Salzmann et al. 2009; Benoit et al. 2009) and few of them (like ice VIII, ice VIII and ice X) are related to high pressure structures (Hirsh \& Holzapfel, 1986). Above 2 GPa of pressure, the structures in general fluctuate in between ice VII and VIII depending up on the temperature (Wolanin et al. 1997). Ice VII has body-centered cubic (bcc) structure (space group symmetry $P n 3 m$ ) and contains two water molecules in a unit cell with disordered hydrogen. Ice VIII on the other hand is formed with I41/amd space group symmetry and tetragonal structure (Yamawaki et al. 2004) which contains eight molecules per unit cell. The atomic arrangement of these two structures is similar except the fact that water molecules are disordered around their centre of mass in bcc structure (Goncharov et al. 1996). The phase transition between them as the function of temperature and pressure is well described by Yamawaki et al (2004). The authors describe ice VIII as the low temperature structure of ice VII.

Ice VIII can also be defined by two interpenetrating Ic ice lattices which are arranged in such a way that the dipole moments of them are equal and opposite, forming the final structure antiferroelectric (Wolanin et al. 1997; Goncharov et al. 1996; Benoit et al. 1998). Detail structural analysis shows that each oxygen atom connects to four other oxygens (among eight nearest oxygens) where it receives protons from two bonded neighbours (one from each) and donates two (one to each) to the remaining two (ice rule) as shown in Fig. 1. Fig. 1 further illustrates that the hydrogen is covalently bonded and received by one of the two oxygens (represented in solid lines) where as it is weakly bonded via hydrogen bond and donated by the other (represented in dashed line). The sum of these two bond-length gives neighbouring oxygen-oxygen separation (O-H..O). 


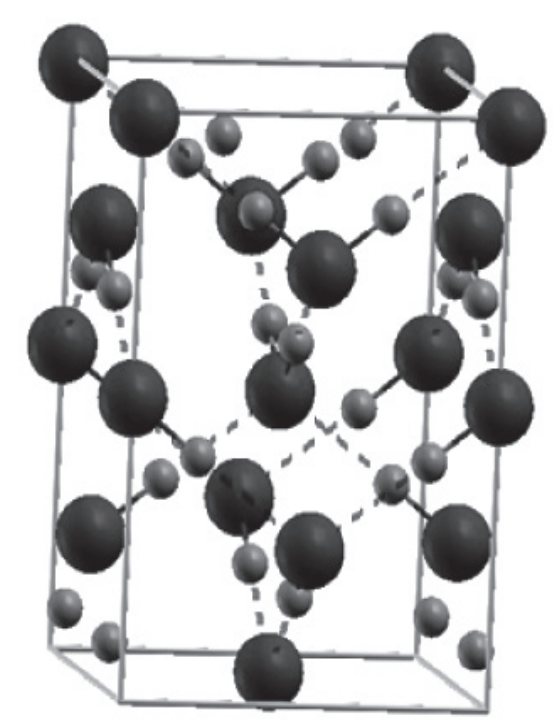

Fig. 1. Atomic arrangement in ice VIII. The large atoms are oxygens and small atoms are hydrogens. The hydrogen binds the bonded neighbouring oxygens and are represented by the combination of solid lines (receive protons) and dashed lines (donate protons), ice rule. The symbol $O$ is the central oxygen which is connected to four of its neighbouring oxygens named as $\mathrm{O}_{1}, \mathrm{O}_{2}, \mathrm{O}_{3}$ and $\mathrm{O}_{4}$. The hydrogen in between $O$ and $O_{1}$ is named as $H_{1}$ and $H_{2}, H_{3}$ and $H_{4}$ are considered on the same way.

On elevating pressure, inter atomic separation between the oxygen atoms (O-H..O) decreases. In addition to the decrease in inter atomic separation between the atoms in the system, hydrogen (proton) between two nearest bonded oxygens comes to midway between them. This process brings initial structure to more symmetrized phase of ice which is called ice X (Loubeyre et al. 1999). The transition pressure for changing ice VIII to symmetric ice $\mathrm{X}$ depends on many factors. Classical treatment for nuclei finds this pressure as $102 \mathrm{GPa}$ while the inclusion of quantum-mechanical effect reduces the pressure significantly and shows the transition pressure as $72 \mathrm{GPa}$ (Benoit et al. 1998). Also the isotropic effect of hydrogen plays influential role for defining pressure required for phase transition.

In the present work, we calculate the changes in atomic positions and cell parameters of ice by taking tetragonal ice VIII as initial structure, in the response of elevating hydrostatic pressure up to $120 \mathrm{GPa}$, and compare the results with the available experimental reports. We also discuss the change in phase of ice VIII to more symmetrized ice $\mathrm{X}$ structure as a function of increase in pressure. We also introduce theoretical details and computational methods used for this work followed by the results, discussion and conclusions.

\section{MATERIALS AND METHODS}

Interaction between a set of nuclei and electrons in a solid can be logically defined by many body problems (Martin, 2004). Time independent Schrödinger equation is the first option to solve these types of many-body interactions and can be expressed as,

$$
H_{t o t} \Psi=E_{t o t} \Psi
$$

with Hamiltonian

$$
\hat{H}_{l o t}=-\frac{\hbar^{2}}{2 m} \sum_{i} \vec{\nabla}_{i}^{2}+\frac{1}{2} \sum_{i \neq j} \frac{e^{2}}{\left|\vec{r}_{i}-\vec{r}_{j}\right|}-\sum_{i, I} \frac{Z_{I} e^{2}}{\left|\vec{r}_{i}-\vec{R}_{I}\right|}-\sum_{I} \frac{\hbar^{2}}{2 M_{I}} \vec{\nabla}_{I}^{2}+\frac{1}{2} \sum_{I \neq J} \frac{Z_{I} Z_{J} e^{2}}{\vec{R}_{I}-\vec{R}_{J} \mid}
$$

In equation 2 , the first, second and third terms of the equation represent kinetic energy of the electrons, electron-electron Coulomb interaction and electronnuclei Coulomb interaction respectively. The fourth and fifth terms on the other hand are the kinetic energy of nuclei and the nuclei-nuclei Coulomb interaction, respectively.

Born-Oppenheimer approximation simplifies the equation by assuming nuclei (heavier particle) stationary with respect to lighter electrons (Cramer, 2004). In this way, the total energy of the system becomes sum of the electron and nuclei energies, and the total wave-function can be separated for electrons and nuclei. Hence total wave-function becomes,

$$
\Psi(\vec{r}, \vec{R})=\Psi_{e}(\vec{r}, \vec{R}) \chi_{(\text {nuсl })}(\vec{R})
$$

Once the electronic and nuclei terms in the Hamiltonian are separated, we first solve the electronic part of the problem which depends on kinetic energy of electrons, electron-electron interaction and also on nuclear coordinates as well. The untouched nuclear part of the problem can be incorporated later on.

$$
\hat{H}_{e}=-\frac{\hbar^{2}}{2 m_{e}} \sum_{i} \vec{\nabla}_{i}^{2}+\frac{1}{2} \sum_{i \neq j} \frac{e^{2}}{\left|\vec{r}_{i}-\vec{r}_{j}\right|}-\sum_{i, I} \frac{Z_{I} e^{2}}{\left|\vec{r}_{i}-\vec{R}_{I}\right|}
$$

The electronic Hamiltonian, $H_{e}$ (defined by equation 4 ), still contains kinetic energy of interacting electrons and coordinates of many-body electrons. However, many body interacting electrons can be transformed to oneelectron equations by using Hartree approximation where the electron moves under the potential of other electrons. Hence the total electron wave function becomes the product of one-particle wave functions. Hartree-Fock assumption is one step advanced and incorporates Pauliexclusion principle by stating total anti-symmetrized wave function in the form of a determinant of one-electron wave functions. In this way the exchange energy, one of the quantum mechanical effects, is included in HartreeFock theory. However, the remaining quantum effects arising from many body interactions, generally defined 
by correlation energy is still outside its coverage.

DFT has become a popular technique to deal with manybody problems in solid state physics/chemistry and material science since last half a century (Sholl \& Steck, 2009; Grosso \& Paravicini, 2000). In DFT we use the information of ground state electron density, instead of all electron wave functions, to calculate the ground state energy of the system. And the ground state energy in principle is enough to calculate many ground state properties of the system.

Hohenberg and Kohn were the people who first defined two theorems to relate ground state energy with the electron density (Hohenberg \& Kohn, 1964). The theorems define ground state energy of the inter-acting electrons as the unique functional of electronic charge density $\rho(\vec{r})$ and furthermore ensure that the functional has its minimum value for the true ground state electron density. KohnSham after a year developed a formalism for the practical applications of DFT in cu rent computational approach (Kohn \& Sham, 1965). The algorithm represents the original interacting electrons into a non-interacting electron moving under the effective potential created by other electrons and nuclei. The total energy functional can now be divided into kinetic energy, Hartree energy, energy due to nuclei and exchange correlation energy as defined by equation (5) in order.

$$
E[\rho(\vec{r})]=T_{o}[\rho]+\frac{1}{2} \iint \frac{\rho(\vec{r}) \rho\left(\overrightarrow{r^{\prime}}\right) d \vec{r} d \overrightarrow{r^{\prime}}}{\mid \vec{r}-\overrightarrow{r^{\prime} \mid}}+\int V_{e x t}(\vec{r}) \rho(\vec{r}) d \vec{r}+E_{x c}[\rho] \ldots(5)
$$

Variation of this equation (5) with respect to density gives a set of one-electron Schrödinger like equations which are called Kohn-Sham equations,

$\left[-\frac{1}{2} \vec{\nabla}^{2}+V_{e f f}(\vec{r})\right] \Psi_{n}(\vec{r})=\varepsilon_{i} \Psi_{n}(\vec{r})$

Where effective potential $V_{\text {eff }}$ is given by,

$$
V_{e f f}(\vec{r})=V_{e x t}+\int \frac{\rho\left(\overrightarrow{r^{\prime}}\right) d \vec{r}}{\left|\vec{r}-\overrightarrow{r^{\prime}}\right|}+V_{x c}
$$

such that $V_{x c}=\frac{\delta E_{x c}[\rho]}{\delta \rho(\vec{r})}$

In equation $7, V_{\text {ext }}$ is the external potential due to nuclei and the remaining electrons and $V_{x}$ is the exchangecorrelation potential. Kohn-Sham equations calculate the Kohn-Sham eigen values $\left(\varepsilon_{i}\right)$, Kohn-Sham orbitals and then electron density. Hartree and exchange correlation energy are the functional of electron density and can be solved in self consistent manner. The kinetic energy of the electrons and energy due to external potential (nuclei) can be incorporated according to the system used. For computational work, we start from the initial charge density by using the information of atomic configurations, calculate the effective potential and then solve the KohnSham equations to find the ground state parameters.

For our purpose density-functional theory calculations were performed by using Quantum-ESPRESSO package (Giannozzi et al. 2009; Scandolo et al. 2005). The electron-ion interaction was taken into account with ultrasoft pseudo potentials. Perdew-Burke-Ernzerhof (PBE) form of generalized-gradient approximation (Perdew et al. 1996) was chosen to describe the exchange and correlation part of the electron-electron interaction.

The unit cell parameters $(a=4.574 \mathrm{~A}$ and $c=6.621 \mathrm{~A})$ of ice VIII containing eight water molecules in tetragonal lattice structure with space group I41/amd were taken from the X-rays diffraction experiment (Yamawaki et al. 2004). Convergence tests of the system were then carried out with respect to lattice parameter $a$ and kinetic energy of wave functions. The parameters of the structure were then finalized accordingly. K-point mesh of $4 \times 4 \times 3$ Monkhrost-pack grids in the first brillouin zone was set according to its reciprocal property in $\mathrm{k}$-space with respect to the geometry in real space. The external parameters as well as internal co-ordinates were relaxed at the selected pressures which are mainly consistent with the experimental values (Yamawaki et al. 2004).

\section{RESULTS AND DISCUSSION}

In this section, we present and discuss the main finding of our calculations and compare them with the previously reported values. We begin the section with defining initial structure which is followed by the analysis on changing structural parameters due to compression. The section finally includes the discussion on change in phase from initial structure of ice to symmetric hydrogen-bonded ice, called "ice X".

\section{Defining initial conditions}

Before calculating the structural and electronic properties/ parameters of any material by using density functional theory (DFT), we need to select a proper building block cell of the sample and its ground state structure. As discussed in introduction section, ice VIII structure of our choice has a tetragonal unit cell. To define the lattice constant corresponding to equilibrated tetragonal unit cell and then its cell parameters, the sample was optimized at varying values of $a$, one of the cell sides (shown in Fig. 2) 


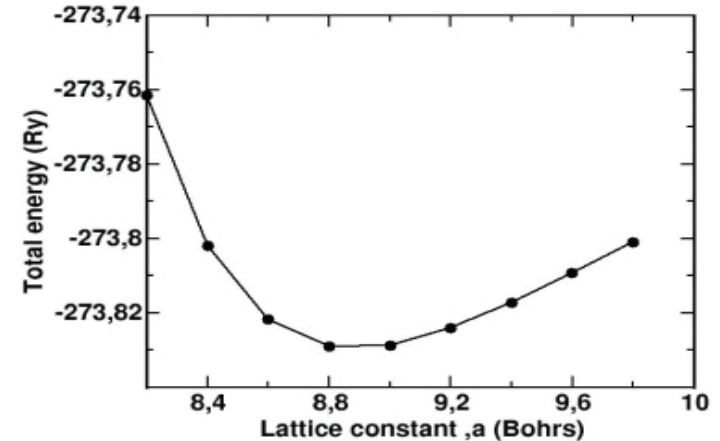

Fig. 2. Total energy variance with lattice parameter (a) of tetragonal ice VIII.

Fig. 2 shows the variation of the total energy of the system with the cell parameter $a$. The energy is seen minimized at around $a=4.66 \AA$ ( 8.8 Bohrs) and this becomes the best choice for two sides $(a, b)$ of the tetragonal unit cell. Within the limit of the intervals selected during the calculations and the number of significant figures, the calculated value lies within $2 \%$ of the experimental observation (Yamawaki et al. 2004). The remaining side of the cell, $c$, was chosen as $(\sqrt{2} \times a)$ according to its standard structure. Further calculations are also performed to see the total energy variance with respect to kinetic energy of wave functions for electrons, as a part of convergence tests (Fig. 3).

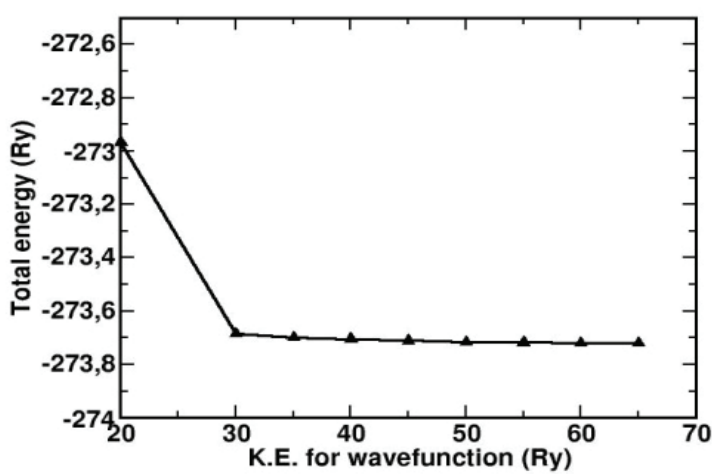

Fig. 3. Total energy variance with kinetic energy of the electron wave-functions. Cut off at $35 \mathrm{Ry}$ is a good approximation in this case.

The total energy variance with respect to the kinetic energy of the wave function, as shown in Fig. 3, has been done to choose the cut off value for the kinetic energy of wave function without much compromising in its accuracy. The wave functions for electrons in momentum space, in principle, are expanded up to infinity for accurate calculations. The expansion however takes a lot of computational cost and not practical to attain. Finally the competition between the accuracy and the computational cost of the system enforces us to compromise somewhere between them and leads to truncate energy for wave functions at some value by taking care of the required accuracy. In Fig. 3, the total energy of the system becomes nearly independent to the kinetic energy of the wave functions when it rises above $35 \mathrm{Ry}$. This makes sense to choose its cut off at $35 \mathrm{Ry}$.

\section{Compression of the structure}

The initial structure of tetragonal ice VIII at $5 \mathrm{GPa}$ is compressed by hydrostatic pressure in the steps of few $\mathrm{GPa}$ up to $120 \mathrm{GPa}$. The initial pressures up to $47.1 \mathrm{GPa}$ are basically chosen in consistent with the previously reported experimental results where as the pressures beyond $60 \mathrm{GPa}$ are selected in the steps of $10 \mathrm{GPa}$.

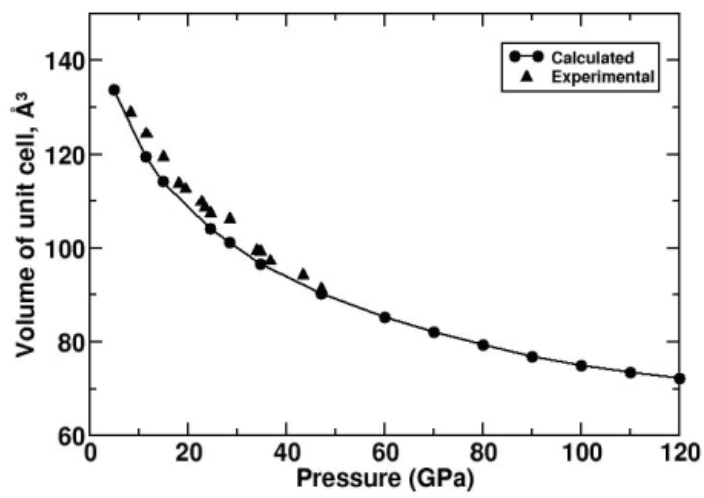

Fig. 4. Pressure dependence of the volume of ice VIII. The black solid line represent our calculated values where as up-triangles represent the experimental values at $87 \mathrm{~K}$

(Yamawaki et al. 2004).

Table 1. Represents the comparison between experimental and calculated unit cell volumes of ice VIII at varying pressures (Yamawaki et al. 2004). The absolute and percentage differences between them (with respect to calculated ones) are also mentioned.

\begin{tabular}{|c|c|c|c|c|}
\hline $\begin{array}{c}\text { Pressure } \\
(\mathrm{GPa})\end{array}$ & $\begin{array}{c}\text { Calculated V } \\
\left(\AA^{3}\right)\end{array}$ & $\begin{array}{c}\text { Experimental } \\
\text { V }\left(\AA^{3}\right)\end{array}$ & $\begin{array}{c}\text { Diff. } \\
\left(\AA^{3}\right)\end{array}$ & Diff. \% \\
\hline 11.5 & 119.404 & 124.34 & 4.936 & 3.969 \\
15 & 114.123 & 119.47 & 5.347 & 4.476 \\
24.5 & 104.099 & 107.42 & 3.321 & 3.091 \\
28.4 & 101.212 & 106.26 & 5.049 & 4.751 \\
34.8 & 96.646 & 99.221 & 2.575 & 2.596 \\
47.1 & 90.299 & 91.279 & 0.980 & 1.074 \\
\hline
\end{tabular}

The hydrostatic pressure applied to the unit cell exerts equal force in all the directions. This obviously compresses the unit cell. Fig. 4 and table 1 show that the compression elevates monotonically with increasing pressure. The comparison of the quantities from the reference experiment and our DFT calculations reflects acceptable range of agreement (maximum difference between them is less than $5 \%$ with respect to the calculations), with slightly higher experimental values. 
One can see the difference in temperatures as the experiments were performed at $87 \mathrm{~K}$ and the counterpart DFT calculations are done at $0 \mathrm{~K}$. It is logical to expect that the increasing temperature enhances volume of the unit cell and inter-atomic separation. A separate work performed by Besson et al (1994) approves the expansion of ice by $3.9 \%$ on elevating temperature from $0 \mathrm{~K}$ to 300 $\mathrm{K}$. It implies that the inclusion of temperature-effect DFT calculations further narrow down the differences. Among the calculated volumes of the unit cell at different pressure, volume at $60 \mathrm{GPa}$ is compressed by $36.39 \%$ comparing to that at $5 \mathrm{GPa}$. In addition to the compression in volume, we report change in cell dimensions $(a \& c)$ of the sample due to growing pressure (Fig. 5).

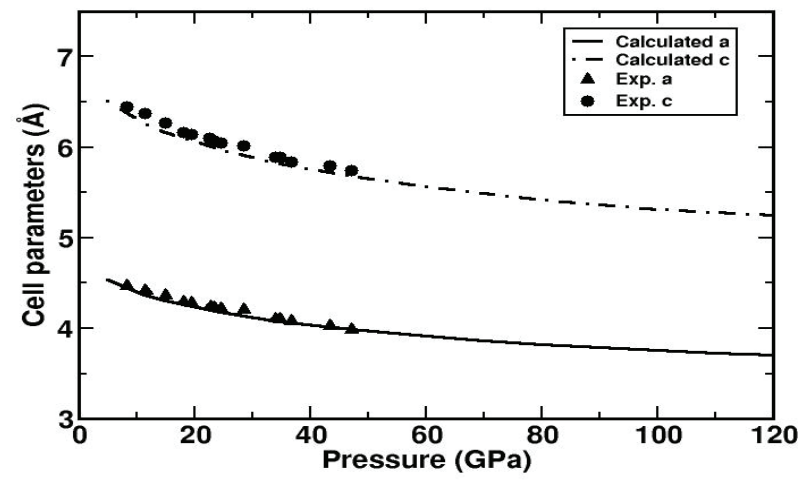

Fig. 5. Pressure dependence of the cell parameters along $a$ and $c$ directions. The calculated values of $a$ and $c$ are represented by black solid lines where as their experimental counterparts are represented by blue circles and green up triangles respectively.

In spite of isotropic pressure acting on any solid body, because of varying structural compactness, it is not necessary that the material should be squeezed by equal proportion in all the directions. Fig. 5 shows almost isotropic compression along $a$ and $c$ directions of tetragonal ice VIII on elevating pressure in the steps of few GPa. This can be quantified by observing calculated values of the unit cell sides, $a$ and $c$, which at $60 \mathrm{GPa}$ are decreased by $13.7 \%$ and $14.59 \%$ respectively, comparing to that at $5 \mathrm{GPa}$. Fig. 5 further shows that within the limit of temperature effect, the experimental cell sides fit well with the present results.

\section{Phase change to ice $X$}

In this section we report the change in bonded oxygenoxygen separation $\left(d_{o-o}\right)$, oxygen-hydrogen separation $\left(d_{O-H}\right)$ and the nature of hydrogen bonding in between the oxygens as a function of pressure (Fig. 6). While decreasing the oxygen-oxygen separation on increasing pressure, $d_{O-O}$ reaches to a critical distance where the position of hydrogen comes to a single minimum potential instead of its fluctuations in between two minima at low pressure structure of ice (ice VIII). This new situation creates a new phase of ice which is known as ice $X$. The ice $\mathrm{X}$ in Fig. 6 can be recognized by the symmetrization of hydrogen bonding in between the oxygens.

Fig. 6 shows an interesting behaviour of $d_{O-H}$ separation as a function of pressure. As we see in Fig. 1, hydrogen joins a pair of oxygen atoms where it is close to one of them by being almost one-third of the total oxygen-oxygen separation comparing to two-third of the distance from its distant counterpart. On elevating pressure, however, its distance from the nearer one increases and from the distant one decreases. Thus, the difference between bond lengths decreases on moving up the compression until hydrogen comes exactly at the mid point of the bonded oxvgens.

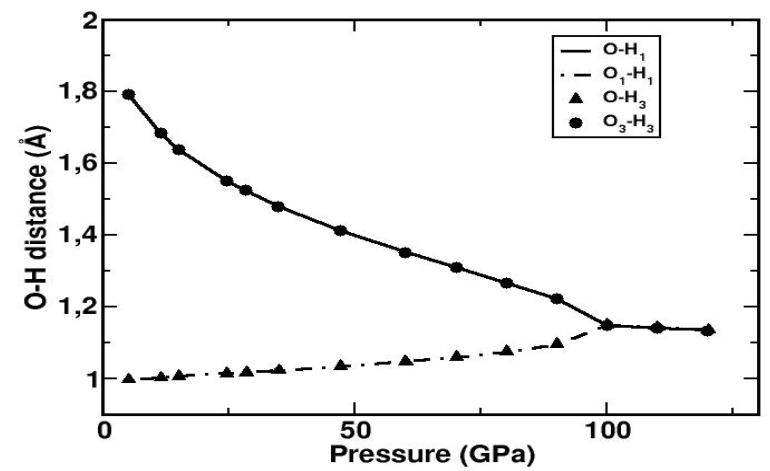

Fig. 6. Pressure dependence of oxygen-hydrogen separation. The symbols $\mathrm{H} 1$ connects oxygens $\mathrm{O}$ and $\mathrm{O}_{1}$, and $\mathrm{H}_{3}$ connects $\mathrm{O}$ and $\mathrm{O}_{3}$ as defined in Fig. 1 .

Fig. 6 also implies that separation between the bonded oxygen atoms $\left(d_{O-O}\right)$ decreases on increasing pressure. This compression (change) in bond-length reaches to $13.98 \%$ at $60 \mathrm{GPa}\left(d_{O-O}=2.40 \mathrm{~A}\right)$ comparing to its initial separation $(2.79 \mathrm{~A})$ at $5 \mathrm{GPa}$. We have also observed the position of the proton and oxygen-oxygen separation, containing the proton in between them, up to $120 \mathrm{GPa}$. The calculations show that the symmetrization of hydrogenbonding which defines the structural transition from ice VIII to ice X, occures at $100 \mathrm{GPa}$. This change in phase of ice into a more symmetrized structure, which is already defined in this section as ice $\mathrm{X}$, is characterized by the oxygen-oxygen separation $d_{O o}=2.30 \AA$. This number is very near to to previous study performed by Wolanin et al. (1997) $\left(d_{O-O}=2.32 \AA\right)$, and others (Loubeyre et al. 1999). The same separation between the oxygen atoms $\left(d_{O-O}=2.32 \AA\right)$ during the symmetrization of ice channels has been observed in our separate calculations for methane hydrate clathrates (Pantha et al. 2014). The transition pressure for the symmetrization of ice by DFT calculations on the other hand has been mentioned as 102 GPa by Benoit et al. (1998). The pressure of course is close enough with our estimation. 


\section{CONCLUSIONS}

Our DFT calculations show that the external (cell sides) and the internal (interatomic separation) parameters of ice, taking ice VIII as initial structure, decrease monotonically on elevating hydrostatic pressure up to $120 \mathrm{GPa}$. The separation between the bonded oxygens at transition pressure $(100 \mathrm{GPa}), 2.30 \AA$, is similar to the other DFT calculations (Benoit et al. 1998) and experimental results (Wolanin et al. 1997). We have also calculated the transition pressure, $100 \mathrm{GPa}$, for changing ice VIII structure to ice X. Further calculations on smaller interval of pressure may precisely predict the transition pressure for symmetrization of hydrogen bonding in high pressure ice structure.

Better $a b$ initio approach may consider nuclei of the system as quantum particles to include the quantummechanical effects on transition pressure and critical oxygen-oxygen separation for the symmetrization of hydrogen bonding. Also the isotropic effects of hydrogen can be addressed by taking an account of deuterium along with the proton of the present work. Magnetic properties and minute study of hydrogen ordering, which defines the boundary between ice VIII and ice VII structures, can be an immediate extension of the present work.

\section{ACKNOWLEDGMENT}

NP acknowledges STEP programme of The Abdus Salam International Center for Theoretical Physics (ICTP) for providing partial support. We also would like to acknowledge Nepal Academy of Science and Technology (NAST) for partial support. Further we acknowledge the partial support from ICTP through office of external activities NET-56.

\section{REFERENCES}

Adhikari N.P, Poudyal, H, Tiwari, A. and Johri, M. 2011. Theoretical and experimental study of hydrogen bonded liquids with water as an example. Journal of molecular liquids 158: 80.

Benoit, M., Bernasconi, M., Focker, P. and Parrinello M. 1996. New high-pressure phase of ice. Phys. Rev. Lett. 76: 2934.

Benoit, M., Marx, D. and Parrinello, M. 1998. Tunneling and zero-point motion in high pressure ice. Nature 392:258.

Besson, J.M., Pruzan, Ph., Klotz, S., Hamel, B., Silvi, B., Nelmes, R.J., Loveday,J.S, Wilson, R.M. and Hull, S. 1994. Variation of interatomic distances in ice VIII to 10 GPa. Phys. Rev. B 49:12540.

Cavazzoni, C., Chiarotti, G.L., Scandolo S., Tosatti, E., Bernasconi, M., and Parrinello, M. 1999. Superionic and metallic states of water and ammonia at giant planet conditions. Science 283:4.

Cramer, C.J. 2004. Essentials of Computational Chemistry: Theory and Models, $2^{n d}$ ed., John Wiley and Sons Ltd., England.
Giannozzi P.; 2009. QUANTUM ESPRESSO: a molecular and open-source software project for quantum simulations of materials, J. Phys.: Condens. Matter 21:395502.

Goncharov, A.F., Struzhkin, V.V., Somayazulu, M.S., Hemley, R.J. and Mao, H. K. 1996. Compression of ice to 210 gigapascals: infrared evidence for a symmetric hydrogen bonded phase. Science 273:218.

Grosso, G. and Parravicini, G.P. 2000. Solid State Physics, Academic Press, London.

Hemley, R.J., Jephcoat, A.P., Mao,H.K., Zha, C.S, Finger, L.W., and Cox, D.E. 1987. Static compression of H2O-ice to $128 \mathrm{GPa}$ (1.28 Mbar). Nature 330: 737.

Hirsch, K.R. and Holzapfel, W.B. 1986. Effect of high pressure on the Raman spectra of ice VIII and evidence for ice X. J. Chem. Phys. 84(5):2771.

Hohenberg, P. and Kohn, W. 1964. Inhomogeneous electron gas. Phys. Rev. 136: B864.

Kohn, W. and Sham, L.J. 1965. Self-consistent equations including exchange and correlation effects, Phys. Rev. 140: A1133.

Kuhs, W.F., Finney, J.L., Vettier, C. and Bliss, D.V. 1984. Structure and hydrogen ordering in ices VI, VII, and VIII by neutron powder diffraction. J. Chem. Phys. 81(8): 3612 .

Lee, M.S. and Scandolo, S. 2011. Mixture of planetary ices at extreme conditions. Nature Comm. 2: 18.

Loubeyre, P., LeToullec, R., Wolanin, E., Han M. and, Hausermann, D. 1999. Modulated phases and proton centering in ice observed by X-ray diffraction up to 170 GPa, Nature 397: 503.

Martin, R.M. 2004. Electronic Structure: Basic Theory and Practical Methods, Cambridge Uni-versity Press, United Kingdom.

Pantha, N., Adhikari, N.P. and Scandolo S., Decomposition of methane hydrate clathrates at high pressure: a density functional theory study, manuscript to be submitted.

Perdew, J., Burke, K. and Ernzerhof, E. 1996. Generalized gradient approximation made sample. Phys. Rev. Lett. 77: 3865 .

Salzmann, C.G, Radaelli, P.G., Mayer, E., and Finney, J.L. 2009. Ice XV: A new thermodynamically stable phase of ice. Phys. Rev. Lett. 103: 105701.

Scandolo, S., Giannozzi, P., Cavazzoni,C., Gironcoli, S. de., Pasquarello, A. and Baroni, S. 2005. First-principles codes for computational crystallography in the Quantum-ESPRESSO package. Z. Kristallogr. 220: 574.

Sholl, D.S and Steck, J.A. 2009. Density Functional Theory: A Practical Introduction, A John Wiley and Sons, Inc., New Jersey.

Sloan E.D, and Koh, C.A. 2007. Clathrate Hydrates of Natural Gases, $3^{\text {rd }}$ ed., CRC Press, California.

Wolanin, E., Pruzan, Ph., Chervin, J.C., Canny, B., and Gauthier, M. 1997. Equation of state of ice VII up to 106 GPa. Phys. Rev. B 56: 5781.

Yamawaki, H., Fujihisa, H., Sakashita, M., Nakayama, A., and Aloki, K. 2004. Powder X-ray diffraction study of the volume change of ice VIII under high pressure. Physica B 344 : 260. 